\title{
Brown Tumor of the Maxilla Revealing Primary Hyperparathyroidism
}

\author{
Madiha Mahfoudhi ${ }^{*}$, Khaled Khamassi², Amel Gaieb Battikh1, Rym Lahiani², Turki Sami', \\ Mamia Ben Salah ${ }^{2}$ \\ ${ }^{1}$ Department of Internal Medicine A, Charles Nicolle Hospital, Tunis, Tunisia \\ ${ }^{2}$ Department of Oto-Rhino-Laryngology, Charles Nicolle Hospital, Tunis, Tunisia \\ Email: ${ }^{*}$ madiha_mahfoudhi@yahoo.fr
}

Received 22 January 2015; accepted 11 April 2015; published 16 April 2015

Copyright $@ 2015$ by authors and Scientific Research Publishing Inc.

This work is licensed under the Creative Commons Attribution International License (CC BY).

http://creativecommons.org/licenses/by/4.0/

(c) ()

\begin{abstract}
Brown tumor or localized form of osteitis fibrosa cystic is a focal lesion complicating hyperparathyroidism. It's rarely revelatory of the clinical feature and corresponds to a mass with partly cystic and partly solid areas. A 65-year-old man, followed up for hypertension, complained of a progressive swelling in the left paranasal part of the face lasting for 3 months. Physical examination revealed a left paranasal swelling of $4 \mathrm{~cm}$ diameter, with a healthy looking skin. CT facial bones found a round formation of $2 \mathrm{~cm}$ involving the left maxilla. He received tumor resection by vestibular pathway. Histological examination showed many giant cells. We then discussed the brown tumor diagnosis. The PTH dosage was high: $645 \mathrm{pg} / \mathrm{mL}$ (normal: 15 - $68 \mathrm{pg} / \mathrm{mL}$ ). The serum calcium was in the superior normal limit $(100 \mathrm{mg} / \mathrm{dL})$. Cervical ultrasound revealed a bottom left parathyroid nodule. A lower left parathyroidectomy was performed. Histological results confirmed parathyroid adenoma. Monitoring was marked by a marked decrease of the PTH serum level then a return to normal values, a normocalcemia and no recurrence of the maxillary tumor with Recoil of 14 months. Brown tumor of the maxilla is rare and should be considered even in absence of hypercalcemia.
\end{abstract}

\section{Keywords}

Hyperparathyroidism, Brown Tumor, Maxilla

\section{Introduction}

Primary hyperparathyroidism is inappropriate secretion of parathyroid hormone (PTH) inducing commonly

"Corresponding author.

How to cite this paper: Mahfoudhi, M., Khamassi, K., Battikh, A.G., Lahiani, R., Sami, T. and Salah, M.B. (2015) Brown Tumor of the Maxilla Revealing Primary Hyperparathyroidism. International Journal of Clinical Medicine, 6, 252-256.

http://dx.doi.org/10.4236/ijcm.2015.64031 
hypercalcemia. It’s frequently latent, especially involving postmenopausal women. Hypersecretion of PTH is due to adenomas in $85 \%$ of all cases. It's classically revealed by hypercalcemia and hypophosphatemia [1]-[4].

Primary hyperparathyroidism induces two main complications: bones and kidneys lesions.

It leads to renal stones formation or nephrocalcinosis corresponding to diffuse parenchymal calcium deposits [1].

Characteristic skeletal disturbances complicating this disease are essentially osseous resorption, bone cysts, brown tumors and generalized osteopenia. These lesions involve more frequently the ribs, clavicles, pelvic girdle, and the mandible. They affect exceptionally the maxilla [2].

About $2 \%$ of the patients develop osteitis fibrosa cystica, and $0.8 \%$ develop Brown tumor. Brown tumor is the localized form of osteitis fibrosa cystic [5]-[7].

An excess of parathyroid hormone can be complicated of brown tumor corresponding to a reactive lesion in the bone. Osteitis fibrosa cystica is a diffuse resorptive process of the bone, secondary to localized rapid osteoclastic bone turnover due to direct effect of PTH. Normal bone marrow elements are replaced by hemorrhage, vascular fibrous tissue, and resultant granulation tissue. Bone expansion results from accumulation of fibrous tissues and giant cells. Brown tumor can be encountered in primary hyperparahyroidism and less frequently in the secondary form. It belongs to giant cells lesions [2].

Less than $5 \%$ of cases are recognized by the presence of brown tumors [5]-[7].

The aim of this study is to remind clinicians that brown tumors should be considered as rare revealing forms of primary hyperparathyroidism.

\section{Case Report}

A 65-year-old man, followed up for hypertension, complained of a progressive swelling in the left paranasal part of the face lasting for 3 months and a pain of the left side of the face and the left eye. He had no history of urinary stones. Physical examination revealed a left paranasal swelling of $4 \mathrm{~cm}$ diameter, ill-defined, hard, painless with a healthy looking skin. He had no motor impairment, normal eyeball movements and visual field.

The biological investigations showed a high level of serum alkaline phosphatase, a normal renal function tests and a low normal phosphorus level of $2.6 \mathrm{mg} / \mathrm{dL}$. The PTH intact level was elevated with a value of $645 \mathrm{pg} / \mathrm{mL}$ (normal range: 15 - $68 \mathrm{pg} / \mathrm{mL}$ ); objectifying the primary hyperparathyroidism. The serum calcium was in the superior normal limit (100 mg/dL).

Renal ultrasonography didn’t reveal urinary stones or nephrocalcinosis.

Total radiography of the skull showed a generalized osteopenia.

A research for multiple endocrine neoplasm types 1 and 2A was negative.

Facial CT facial found a round formation of $2 \mathrm{~cm}$ involving the left maxilla (Figure 1 ). The normal residual sinus was displaced and the left nasal cavity was reduced.

In order to avoid facial deformity and visual damage, a tumor resection by vestibular pathway was performed. The tumor was brownish and firm in texture.

Histological examination showed many osteoclastic giant cells with cellular and vascular stroma and small fragment of reactive bone, which suggested central giant cell granuloma, or giant cell tumor of the bone.

The normocalcemia in our case was a cause of diagnosis lateness, so the hyperparathyroidism was not evoked in the beginning.

The discovery of the hyperparathyroidism in our patient was done after the resection of the maxilla tumor. The histological results were in favor of a brown tumor. Thus, a dosage of PTH was performed confirming the diagnosis.

Cervical ultrasound revealed a bottom left parathyroid nodule.

A lower left parathyroidectomy was performed. Histological examination concluded to a parathyroid adenoma composed essentially of chief cells with a glandular pattern in places.

Monitoring was marked by a decrease of the PTH serum level then a return to normal values, a normocalcemia and no recurrence of the maxillary tumor or the parathyroid adenoma with recoil of 14 months.

\section{Discussion}

Hyperparathyroidism, corresponding to a high level of PTH, leads commonly to renal and skeletal complications. The excess of PTH induces osteitis fibrosa cystic or brown tumor marked by a diffuse osseous resorption 


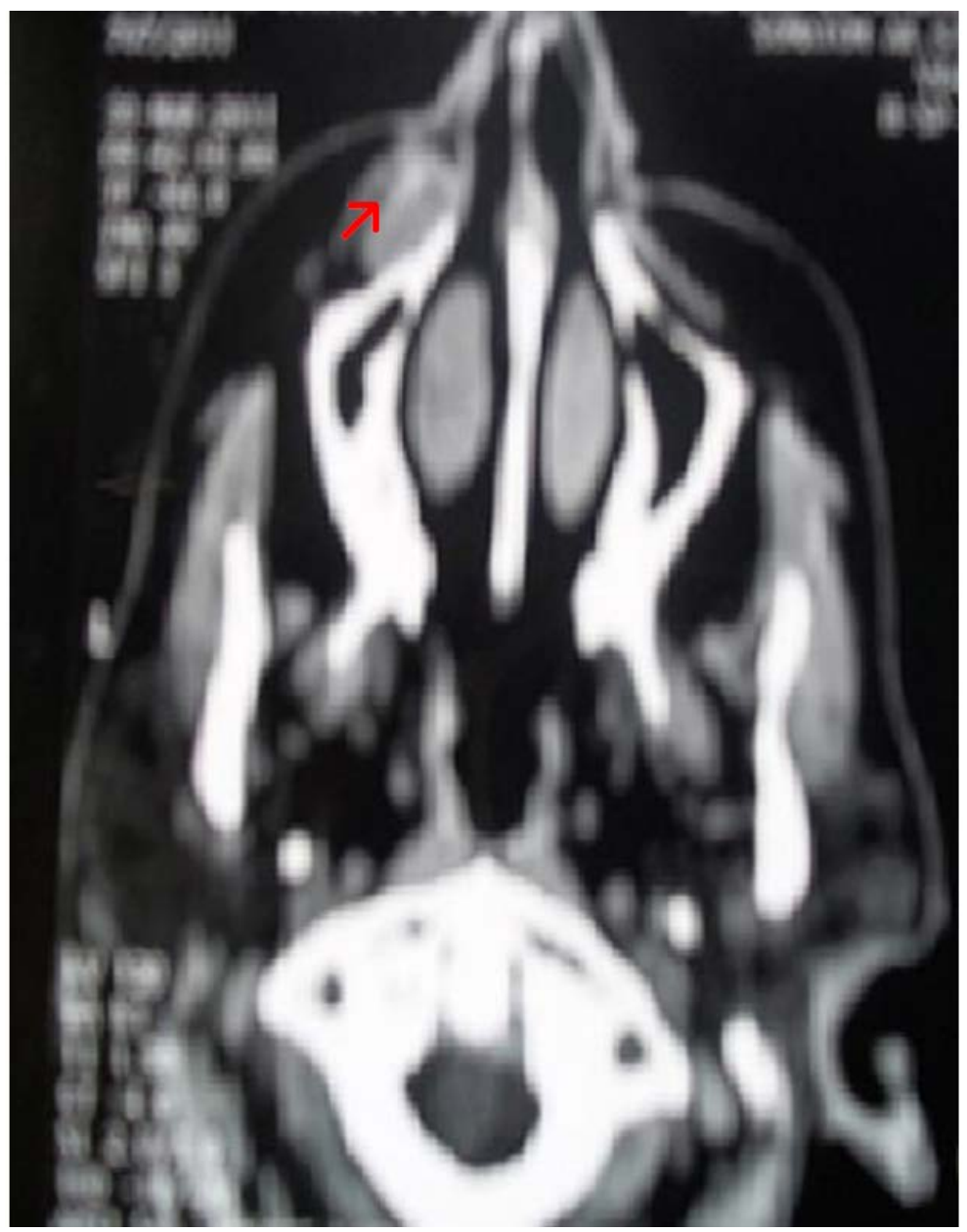

Figure 1. Facial CT (axial section): a round formation of $2 \mathrm{~cm}$ involving the left maxilla.

process of the bone. It can be seen in primary and secondary hyperparahyroidism. A prolonged elevated PTH level causes fibrous and osteoclastic reactions in the skeleton [1]-[5].

An imbalance between bone formation and resorption manifests as bone lysis with fibrous reaction and cortical thinning. These lesions are classically located in phalanges and lamina dura. A focal demineralization and generalized osteoporosis can be encountered [2] [3].

Brown tumor is related, in the majority of cases to primary hyperparathyroidism:

-In $80 \%$ of cases: parathyroid adenoma.

-More rarely hyperplasia.

-Exceptionally parathyroid carcinoma.

Achieving jaw remains, however unusual.

Secondary hyperparathyroidism in chronic kidney disease is more responsible for bone demineralization diffuse, rarely brown tumor.

A case of brown tumor on the maxilla associated with primary hyperparathyroidism was presented by Guney et al. They reported that, currently, the mandible is the most frequently involved bone in the head and neck region and maxillary lesion is extremely rare. Primary hyperparathyroidism is most often discovered incidentally during a blood test showing asymptomatic hypercalcemia [6].

At clinical onset of primary hyperthyroidism, the most common symptoms are due to hypercalcemia (pain due to urinary lithiasis, polyuria, gastrointestinal and neurological disorders) while signs due to brown tumors are not common and rarely revelatory.

Morano et al. discussed a case in which the patient showed recurrent mandibular brown tumors as initial clin- 
ical symptoms of primary hyperparathyroidism [7].

The discovery of the primary hyperparathyroidism from a brown tumor is rare or exceptional [8].

The location of the maxilla brown tumor is rare, but may be the only witness to the parathyroid hyperfunction.

The clinical feature shows an aspect common to other tumors and pseudotumors of the jawbone. The differential diagnosis comprises central giant cell granuloma, giant cell tumor of bone, and osteitis fibrosa cystica (brown tumor) in combination with hyperparathyroidism.

Through two cases of maxillary brown tumor, reported by Thorwarth et al., the reparative giant cell granuloma represents an important differential diagnosis of brown tumor; histological examination or radiological results are insufficient to show difference with the other tumors. Complete information about the clinical signs and the endocrinological status suggest the diagnosis [9] [10].

The imagery found nonspecific osteolysis mimicking tumoral and metastatic bone disease aspects. The most common appearance is that of bone lysis with non-specific limits resulting in blowhole cortical.

The brown tumor should be considered as a rare differential diagnosis of a facial bone lysis [9] [10].

Hypercalcemia is classically revelatory of primary hyperthyroidism complicated of brown tumor. But, it's not pathognomonic, since it can be due to metastatic lesions. In case of maxillary process, only a high serum PTH level permits to evoke a brown tumor. Thus, the dosage of the PTH is the key to diagnosis.

According to Stolovitzky, et al., giant cell lesions of the maxilla present with distinct histological and clinical features; therapeutic modalities, prediction of clinical behavior are controversial [11].

Fancello et al. showed, through 17 cases of cranio-facial giant cell reparative granuloma called also giant cell lesion, the difficulty in differential diagnosis and therapeutic surgical decision [12].

Cervical Imaging (Echography, CT, MRI, Scintigraphy Sestamibi) is used to investigate the causal parathyroid lesion and to determine appropriate therapeutic approach [9] [10] [13].

There is no common opinion as to the therapeutic management of bony lesion once parathyroidectomy has been carried out.

The treatment is based on the excision of the parathyroid lesion (excision of adenoma, subtotal parathyroidectomy in case of hyperplasia).

Isolated parathyroidectomy avoids operating brown tumors which could regress after removal of the parathyroid lesions.

This was not the case in our patient, where the diagnosis was evoked after excision and histological examination of the maxillary tumor. The discovery of giant cell lesions incited us to dose serum PTH level allowing the confirmation of hyperparathyroidism.

In a series of 22 patients presenting facial brown tumor a spontaneous progressive regression were mentioned in all the cases. In 18 cases, regression was total in a mean period of 10 months. Whereas, two patients had presented partial regression [14].

According to this study, Reséndiz-Colosia et al. showed that the bony lesions had regressed spontaneously, partially or completely after successful parathyroidectomy. However, in case of persistent, deforming or symptomatic form, surgical excision of the brown tumor should be performed [14].

Prognosis depends on diagnosis delay and rapid parathyroidectomy.

\section{Conclusions}

Giant cell lesion of the jaws should systematically seek primary hyperparathyroidism by doing a calcium and phosphate serum levels and a dosage of PTH.

The imagery is valuable input for the diagnosis and monitoring of brown tumor. It also allows for the etiological diagnosis by detecting parathyroid lesion. The rapid treatment of the hyperparathyroidism prevents brown tumors formation or permits commonly spontaneous regression of these tumors if already formed.

\section{Disclosure Statement}

The authors have nothing to disclose.

\section{References}

[1] Herowitz, M., Wishard, J.M., Need, A.G., Morris, H.A. and Nordin, B.E. (1994) Primary Hyperparathyroidism. Clinics in Geriatric Medicine, 10, 757-772. 
[2] Parisien, M., Silverberg, S.J., Shane, E., Dempster, D.W. and Bilezikian, J.P. (1990) Bone Diseases in Primary Hyperparathyroidism. Endocrinology Metabolism Clinics of North America, 19, 18-34.

[3] Mundy, G.R., Cove, D.H. and Fisken, R. (1980) Primary Hyperparathyroidism: Changes in the Pattern of Clinical Presentation. Lancet, 1, 1317-1320. http://dx.doi.org/10.1016/S0140-6736(80)91783-3

[4] Owens, B.B. (2009) A Review of Primary Hyperparathyroidism. Journal of Infusion Nursing, 32, 87-92. http://dx.doi.org/10.1097/NAN.0b013e318198d483

[5] Rosenberg, E.H. and Guralnick, W.C. (1962) Hyperparathyroidism. A Review of 220 Proved Cases with Special Emphasis on Findings in the Jaws. Oral Surgery, 157, 82-93.

[6] Guney, E., Yigitbasi, O.G., Bayram, F., Ozer, V. and Canoz, O. (2001) Brown Tumor of the Maxilla Associated with Primary Hyperparathyroidism. Auris Nasus Larynx, 28, 369-372. http://dx.doi.org/10.1016/S0385-8146(01)00099-2

[7] Morano, S., Cipriani, R., Gabriele, A., Medici, F. and Pantellini, F. (2000) Recurrent Brown Tumors as Initial Manifestation of Primary Hyperparathyroidism. An Unusual Presentation. Minerva Medica, 91, 117-122.

[8] El Abdi, B., Berrkia, I., Mohssine, A., El Hassani, M.R., El Quessar, A., Chakir, N., et al. (2006) Tumor of the Maxilla Presenting as Primary Hyperparathyroidism. Journal of Radiology, 87, 1705-1707. http://dx.doi.org/10.1016/S0221-0363(06)74151-8

[9] Thorwarth, M., Rupprecht, S., Schlegel, A., Neureiter, D. and Kessler, P. (2004) Central Giant Cell Granuloma and Osteitis Fibrosa Cystica of Hyperparathyroidism. A Challenge in Differential Diagnosis of Patients with Osteolytic Jawbone Lesions and a History of Cancer. Mund-, Kiefer- und Gesichtschirurgie, 8, 316-321. http://dx.doi.org/10.1007/s10006-004-0556-6

[10] Lehnerdt, G., Metz, K.A., Krüger, C. and Dost, P. (2003) A Bone-Destroying Tumor of the Maxilla. Reparative Giant Cell Granuloma or Brown Tumor? HNO, 51, 239-244.

[11] Stolovitzky, J.P., Waldron, C.A. and McConnel, F.M. (1994) Giant Cell Lesions of the Maxilla and Paranasal Sinuses. Head Neck, 16, 143-148. http://dx.doi.org/10.1002/hed.2880160207

[12] Fancello, A., Caradonna, L. and Tullio, A. (1998) Giant-Cell Reparative Granuloma (GCRG). A Review of the Literature, the Present State of Knowledge and Therapeutic Suggestions. Minerva Stomatologica, 47, 399-407.

[13] Khochtali, H., Ach, K., Jlidi, R., Bouhaouala, H., Ben Gaid Hassine, N., Essakly, M.K., et al. (1991) Bilateral Brown Tumor of the Jaw. A Propos of a Case. Revue de Stomatologie et de Chirurgie Maxillo-Faciale, 92, 116-119.

[14] Reséndiz-Colosia, J.A., Rodríguez-Cuevas, S.A., Flores-Díaz, R., Juan, M.H., Gallegos-Hernández, J.F., BarrosoBravo, S., et al. (2008) Evolution of Maxillofacial Brown Tumors after Parathyroidectomy in Primary Hyperparathyroidism. Head Neck, 30, 1497-1504. http://dx.doi.org/10.1002/hed.20905 